\title{
Proceedings of Society of the British Neurological
}

\section{Surgeons}

\section{The 106th meeting was held in Swansea, 19-20 September 1985}

\section{MYCOTIC CEREBRAL ANEURYSM}

PJE Wilson (Swansea)

Bacterial endocarditis is still a common disease with a high mortality. It was noted that its clinical setting had changed in that it complicated the presence of prosthetic as well as naturally-diseased heart valves. Although mycotic cerebral aneurysm is a relatively rare cause of death or disability in endocarditis, between 1978 and 1985 the author had four patients with a mycotic aneurysm. These patients were just over $1 \%$ of the total number of cases of cerebral aneurysm. All were adult males. Each presented with massive intracerebral haemorrhage. Three patients had a solitary aneurysm and one patient had two aneurysms. All the aneurysms were on peripheral branches of the middle cerebral artery. In two instances, including the fatal case, it seemed likely that associated embolic ischaemia in other branch territories had compounded the purely haemorrhagic brain damage. All patients underwent emergency clot evacuation. The responsible aneurysm was clipped in two cases, and wrapped in the other two. One unruptured aneurysm later healed spontaneously. Three patients survived their haemorrhage and made good neurological recoveries. All presented difficult simultaneous problems of cardiovascular management.

TREATMENT OF RAISED INTRACRANIAL PRESSURE FOLLOWING SEVERE HEAD INJURY

JRS Leggate, NM Dearden and JD Miller (Edinburgh)

The treatment of raised intracranial pressure (ICP) following severe head injury may include evacuation of intracranial mass lesions, artificial ventilation, drainage of CSF and also the use of specific drugs. The authors noted that barbiturates have been used for more than a decade, but that there were many doubts about their efficacy and safety. They therefore had monitored the effects of bolus doses of gammahydroxybutyrate, (GHB, $60 \mathrm{mg} / \mathrm{kg}$ body weight), a short acting anaesthetic agent. They had observed ICP, systemic blood pressure (SBP) and central venous pressure (CVP) and compared the results with the effects of bolus doses of sodium thiopentone (STP $4 \mathrm{mg} / \mathrm{kg}$ ). The studies were performed on 15 patients whose ICP was $>25 \mathrm{mmHg}$ following a severe head injury. ICP was monitored from the subdural space using the Gaeltec ICT/b transducer. The validity of the measurements was verified by comparison with concomitant measurements of ICP from intraventricular and subdural catheters or a subdural screw. The two drugs (GHB and STP) produced similar falls in ICP but the duration of change was longer after GHB $(64 \pm 13.7$ min Mean \pm SD; STP $22 \pm 9 \cdot 3 \mathrm{~min})$. Mean SBP fell equally with both drugs (GHB $17 \pm 15 \mathrm{mmHg}$, STPP $15 \pm 6 \mathrm{mmHg}$ ), and overall cerebral perfusion pressure (CPP) was preserved. There was no correlation between the reductions SBP and CVP. The conclusion of the study was that gammahydroxybutyrate might have advantages over barbiturates in the reduction of ICP.

DIAGNOSIS AND MANAGEMENT OF DELAYED TRAUMATIC INTRACRANIAL HAEMATOMA D Gentleman, F Nath, P Macpherson, L Murray, G Teasdale (Glasgow)

A delayed traumatic intracranial haematoma is diagnosed when a head injured patient develops a new lesion in a part of the brain that appeared normal on a previous CT scan. The authors observed that although CT scanning had improved the detection of such lesions, their chinical importance was still unclear, in particular how often they were sufficiently "significant" to need removal. They had reviewed all 888 cases of traumatic intracranial haematoma managed in their unit between 1979 and 1983 inclusive, and identified and studied the 23 patients $(2 \cdot 6 \%)$ in whom repeat CT scanning demonstrated a delayed haematoma. None of the 23 patients had a normal CT scan when admitted to the neurosurgical unit, but only eight had immediately undergone craniotomy for evacuation of a haematoma. Intracranial pressure (ICP) was monitored in 14 patients and in eight patients an increasing ICP had led to rescanning at a mean of 31 hours (S $>$ E $> \pm 7.8$ hours) after admission to $\bar{\sigma}$ the neurosurgical unit. The other 15 patients were re-scanned because of either neu- $\mathbb{D}$ rological deterioration or a persistent failure to improve and this occurred at a mean of $82 \mathrm{\omega}$ hours (SE $\pm 18 \cdot 3$ ) after admission. The new $\overrightarrow{0}$ lesion shown on the repeat CT was evacu- ated in nine $(39 \%)$ of the 23 patients and in $\vec{\omega}$ each case rescanning had been done within 48 hours of admission to the neurosurgical unit (mean 30 hours, $S E \pm 18.0$ hours). InO the other 14 patients, diagnosed at a mean of $c$ 86 hours (SE $\pm 19 \cdot 2$ ) from admission, the new haematoma was felt not to be contributing to the patient's condition and therefore was not evacuated. The mortalityo was $33 \%$ and $29 \%$ in the operated and nonoperated groups respectively. Morbidity among survivors was higher in the operatgdo group. The authors concluded that in the extensive experience the development of $\vec{a}$ "delayed" haematoma large enough to merit evacuation was uncommon and had. occurred only in patients whose initial $\mathrm{CT}_{\mathrm{O}}$ scan already had been abnormal. Significant lesions were always diagnosed within $48 \bar{\partial}$ hours of admission to the neurosurgical unit. They emphasised the value of ICP $\mathbb{D}$ monitoring which had identified sooner than clinical assessment the patients with a음 significant haematoma.

RADICAL LASER SURGERY IN THE TREATMENT OF GLIOMAS

G Brocklehurst, Hull

The purpose of this report was to examine $\frac{0}{3}$ the possibility that the use of a surgical laser added benefits to the therapy of gliomas. One hundred and forty eight patients were

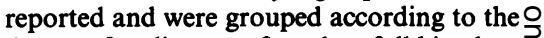
degree of malignancy found on full histolog- $D$ ical examination, the extent of tumouro removal, and if the carbon dioxide (Sharplan 733) surgical laser had been employed. $\mathcal{O}$ Overall the results showed a slight beneficial ${ }_{N}$ effect on the crude survival curve of patients? in whom radical surgery was accompanied $\omega$ by the use of the surgical laser. However a 
higher proportion of low malignancy tumours had been treated with the laser. When this was taken into account it was found that the use of the surgical laser had a negligible effect upon the survival curves for either the patients with a high malignancy tumour or the smaller numbers of patients with a low malignancy lesion.

Table Results of operation on glioma

\begin{tabular}{llrlr}
\hline Malignancy & Low & \multicolumn{3}{l}{ High } \\
Use of laser & No & Yes & No & Yes \\
\hline Survivors at 1 year & 8 & 19 & 14 & 7 \\
Non Survivors & 1 & 6 & 50 & 25 \\
\hline
\end{tabular}

It was concluded that the degree of malignancy of the removed tumour was a far more important factor in determining the length of survival than the addition of the laser to the surgical treatment. Also, that the laser had not facilitated more radical operation or better survival in patients with a relatively low malignant but somewhat deep and inaccessible glioma.

SUBARACHNOID HAEMORRHAGE AND TOTAL CIRCULATING BLOOD VOLUME: METHODOLOGICAL ASPECTS

RJ Nelson, J Roberts, D Ackery, V Walker, JD Pickard, Southampton

The authors pointed out that patients in whom circulating blood volume falls following subarachnoid haemorrhage may be at greater risk of developing cerebral ischaemia, particularly if they have angiographically demonstrable vasospasm. They noted that there was not a direct method for determining the total circulating blood volume (TCBV) of patients, but that there was generally acceptance that the sum of the simultaneously estimated red cell volume (RCV) and plasma volume (PV) was virtually identical to the TCBV. However the simultaneous measurements of RCV and PV was technically demanding and time consuming and might not be appropriate to the management of critically ill patients or patients in whom repeated measurements of blood volume are required. The use of either RCV or PV measurements alone to estimate TCBV made assumptions regarding the constancy of the total body haematocrit/venous haematocrit ratio $(\mathrm{Hb} / \mathrm{Hv})$ in subarachnoid haemorrhage. Moreover, circulating blood volume was usually expressed in terms of body weight but this was a poor predictor of TCBV, which was more closely related to lean body mass and in turn to total body water (TBW). The authors had measured TBW, $\mathrm{RCV}, \mathrm{PV}$ and $\mathrm{Hb} / \mathrm{Hv}$ ratios in 10 consecutive patients with a subarachnoid haemorrhage. The results showed that the commonly accepted value for $\mathrm{Hb} / \mathrm{Hv}$ in haematological practice was not appropriate for subarachnoid haemorrhage patients.

CIRCULATORY ARREST UNDER DEEP HYPOTHERMIA USING CARDIO/PULMONARY BYPASS IN THE MANAGEMENT OF DIFFICULT TYPES OF ANEURYSMS

PG Richards, JM Rice Edwards, London

The authors reported eight patients who had a difficult intracranial aneurysm, either a giant aneurysm or a basilar aneurysm who had undergone operation under deep hypothermia with periods of compete circulatory arrest. Cooling was initiated and controlled by femoro-femoral cardio-pulmonary bypass. Circulatory arrest had allowed safe dissection of the aneurysms without fear of rupture and, by draining some circulating volume into the bypass pump, tension within the aneurysm was reduced to facilitate clipping. They considered results to have been encouraging and that hypothermia under cardio-pulmonary bypass was a useful technique to have available in the operative management of difficult aneurysms.

INTRAOPERATIVE ELECTROCOCHLEOGRAPHY AS A MEANS OF MONITORING HEARING DURING ACOUSTIC NEUROMA SURGERY

P Bentivoglio, D Prasher, L Symon, A Cheesman, London

The ultimate goal in acoustic neuroma surgery is to leave the patient normal with no neurological deficit but often hearing is not preserved. The authors' view was that this may be the result of direct cochlear nerve injury or secondary to some other cause such as cochlear ischaemia due to damage to the artery of the internal auditory meatus. They reported a technique of monitoring intraoperatively the cochlear microphonics and the N1 potential, which they hoped might contribute to preservation of hearing. Seven patients had been studied. Several technical problems had been encountered initially. The active electrode, which was placed on the medial promontory of the middle ear, slipped off this convex surface with subsequent loss of the evoked potential. Fluid (CSF or blood) filled the middle ear via the mastoid air cells and damped down a click stimulus. There had not been complications that could directly be attributed to the placement of the transtympanic active electrode onto the medial promontory. Eventually they were able to achieve a very rapid evaluation of cochlear and cochlear nerve function during surgery. $Z$ Good traces of the electrocochleogram were $\stackrel{D}{\subseteq}$ obtained every 5-10 seconds and the information was fed back immediately to the surgeon. Of the seven cases operated on inco this series, six had anatomical preservation 7 of the cochlear nerve. Only two cases had the N1 potential preserved, and in neither $\frac{\rho}{?}$ was there a recordable wave $V$ on the ABEPs at the end of surgery. All cases with no N1 at the end of surgery were deaf after operation. In the two cases with a preserved $\stackrel{\vec{P}}{\rightarrow}$ $\mathrm{N} 1$ at the end of surgery, one retained nor-믐 mal hearing while the other patient was $\frac{}{0}$ totally deaf. The authors suggested that there were two main benefits of monitoring $\vec{\Phi}$ the electrocochleogram and the ABEPs intraoperatively. (1) They obtained rapid के assessment of cochlear and cochlear nerve $\overrightarrow{-}$ function. (2) From changes in electrical ${ }_{-}^{\circ}$ responses they could deduce the site of dam- $\vec{\omega}$ age to the auditory pathway, and would modify operative technique in order to avoid future occurrences.

EFFECTS OF DEXAMETHASONE AND MANNITOL ON CEREBRAL OEDEMA QUANTIFIED BY MAGNETIC RESONANCE IMAGING

BA Bell, HL MacDonald, DM Kean, MA Smith, GH Barnett, JD Miller JJK Best, Edinburgh

This study used magnetic resonance imaging $\frac{7}{0}$ to look at the vasogenic oedema associated with blood brain barrier breakdown around $\overrightarrow{0}$ a cerebral tumour. The authors drew attê tion to the relation between the $T 1$ parame-. ter of a MR image and both the quantity of water in the tissue, and the degree to which $\Sigma$ it is bound. Cerebral oedema shows clearly on magnetic resonance images. Tumour and surrounding oedematous brain had been $\frac{}{\varnothing}$ imaged in a series of 15 patients and T1 mea- $\varrho$ surements made. All patients had undergone $\overline{\bar{O}}$ operation within 24 hours of imaging, when 3 tumour and abnormal peritumoural brain $\supset$ had been excised. The specimens were rapidly sectioned in the same plane as the mag netic resonance images, and samples of peritumoural tissue had been taken to? correspond with areas where $\mathrm{T} 1$ was mea- $\frac{\sigma}{3}$ sured on the pre-operative image of the brain. The water content of these samples 0 had been estimated indirectly by microgravimetry, and directly by wet and dry을 weighing. A high correlation $(r=0.91,(p<>$ $0.01)$ has been found between $T 1$ and brain $\frac{D}{0}$ water in the excised tissue, and this allowed in vivo brain water to be estimated from the $N$ T1 values in the image of the brain. A fur-ther series of seven patients had been studied 0 before and after the administration of dexamethasone, and during the intravenous 
infusion of a bolus of $20 \%$ mannitol. T1 measurements from the images had shown that dexamethasone did not reduce peritumoural oedema but it did change the NMR characteristics of the tumour itself. The water content of tumour and of the oedematous peritumoural white matter had fallen when a mannitol bolus was given, without dehydrating the normal surrounding brain. They considered that the technique was a useful new tool in cerebral oedema research.

HOW DO WE MANAGE RUPTURED INTRACRANIAL ANEURYSMS? REPORT OF A SURVEY OF BRITISH NEUROSURGEONS

H Marsh, RS Maurice-Williams, KW Lindsay, London

All the consultant neurosurgeons in the British Isles were sent a questionnaire in which they were asked about various aspects of their management of patients with subarachnoid haemorrhage and ruptured intracranial aneurysms. 107 questionnaires were sent out and 96 were returned completed. The number of cases of subarachnoid haemorrhage admitted per surgeon per year ranged from estimates of between 11 and 15 up to between 55 and 60 . The majority admitted between 25 and 50 patients with subarachnoid haemorrhage per year. There were no neurosurgeons who did not operate on intracranial aneurysms and $85 \%$ of surgeons carried out between 10 and 30 operations per year. There were considerable variations in admission policy. Forty nine surgeons had no age limit for accepting patients but only 30 surgeons admitted all cases of subarachnoid haemorrhage as soon as they were referred irrespective of clinical condition. Only 31 surgeons used antifibrolytic treatment before surgery; and only 16 surgeons attempted to control the blood pressure before operation. There were wide differences and no uniformity of practice with respect to the timing of operation and selection of patients for surgery. The only aspect on which the majority of correspondents agreed was that 65 surgeons would operate on a patient admitted with a depressed conscious level only when the conscious level had recovered to normal. There were also considerable differences over various aspects of the technique of surgery; 88 of the respondents used the operating microscope routinely and only four surgeons used no magnification at all. Indirect operations, such as carotid artery ligation, appeared to be little used. Eighty four surgeons used clipping of the neck of the aneurysm as the method of choice and no surgeons used wrapping along. Nineteen neurosurgeons, drawn mainly from among the more senior ranks of the specialty, stated that they would not operate on a nonruptured aneurysm. There appeared to be wide differences in the way in which patients were managed post-operatively with no general agreement on the use of anticonvulsants or steroids, and a number of different regimens employed to treat delayed postoperative ischaemia. The results of this survey appear to show that at the present time, there remain wide differences of opinion as to how patients with subarachnoid haemorrhage should be managed and these differences probably reflect the fact that the value of many aspects of treatment remains controversial and undecided.

EVALUATION OF "NOTIONAL CEREBRAL TRANSIT TIME PARAMETRIC IMAGING" IN PATIENTS SUFFERING SUBARACHNOID HAEMORRHAGE RW Gullan, JD Miller, M Nimmo, MV Merrick, Edinburgh

This study concerned the assessment of cerebral perfusion in patients with subarachnoid haemorrhage using radionuclide cerebral perfusion scintigraphy. The principle employed was that the shape of the dye dilution curve obtained during the first transit of any non-metabolised tracer through any organ could be fitted by a gamma-variate function. A mathematical model had been derived, giving a possible physiological explanation to the constants which could be calculated by fitting the experimentally derived curves. Using a standard gamma camera/minicomputer system, an iv bolus of $750 \mathrm{MBq}$ sodium pertechnetate, and a $32 \times$ 32 matrix, approximately 250 curves were obtained over the cerebral hemispheres in 50 seconds. The curves for each point were analysed to provide a "notional cerebral transit time" which was displayed using a colour scale indicating time. Fifty four studies were performed in 16 patients suffering from subarachnoid haemorrhage. Neurological status and angiographic findings were assessed. Image symmetry was expected and found in 42 , and also in two where asymmetry was not expected. Asymmetry was expected in 12 and present in 10. Notional transit times tended to be symmetrically prolonged in drowsy patients with no lateralising deficit. The authors concluded that the technique was simple, requiring only 50 seconds imaging and using a mobile gamma camera could be performed at the bedside so that it might be useful in assessment of patients suffering from subarachnoid haemorrhage.
INTRACRANIAL VASOSPASM IN SUBZ ARACHNOID HAEMORRHAGE Although cerebral artery spasm has long been considered a major factor in the out come after subarachnoid haemorrhage it haso been extremely difficult to assess. The authors had used spectrally analysed pulsed Doppler ultrasound with on-line analysis op the waveforms to assess spasm in the intra cranial vessels of 28 patients with a ruptured intracranial aneurysm. Ultrasound meas surements were made daily as also were cereo bral blood flow (CBF) measurement usingo the 133 xenon inhalation technique. Patients were divided into high $(n=15)$ and low $(n=13)$ risk groups (predictively poor and good outcomes) at their initial clinicafs assessment and on the appearance of their CT scan. Outcome was assessed as poor of good. The CBF results showed that there was not a significant difference in CBFS when the initial risk group or final outcome were considered. Using Doppler ultrasounष्ठ they had observed significant changes ini fmax over the course of minutes. A total of 24 vessels demonstrated high peak fre quencies (fmax). At angiography 15 vessels demonstrated spasm, one of which had nor-o mal fmaxs. Overall there were no differences in the fmaxs between the left and right sices.or between the side of bleed and the other side. In high risk patients, the fmaxs were slightly higher, peaking around day $0 \overrightarrow{0}$ Fmax also peaked around day 10 in patiefirso with a poor outcome, particularly in low risk patients with a poor outcome. The authors concluded that they had found Transcranial Doppler ultrasound to be sensitive and reliable tool and that the techn nique could have good clinical applications $\frac{\mathrm{O}}{\mathrm{O}}$

EXTRACELLULAR CALCIUM AND CALCIUM ANTAGONISTS IN THE IN VITRO HUMAND

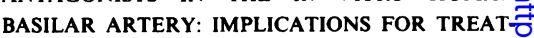
MENT OF CEREBRAL ARTERIAL SPASM SM Marks, AN Jha, RH Lye, ET Whalley, Manchester

The authors commented that although the aetiology of cerebral arterial spasm was. probably multifactorial, it is assumed that all substances involved activated calcium channels, so that calcium antagonists pro-o vide a rational treatment. Their study investigated the role of extracellular calcium and two calcium antagonists, Nimodipineand Diltiazem, on the spasmogenic actions of various substances. They employed basilar arteries obtained postmortem and prepared as described previously. Concen $\widetilde{\omega}$ tration effect curves were produced to potas 
sium chloride (KC1), 5-hydroxytryptamine (5-HT), PGF $2 \mathrm{a}$, Angiotensin II (AII) noradrenaline (NA), U-46619 (a thromboxane $\left(A_{2}\right.$ mimetic), vasopressin and $P E_{2}$ in normal and calcium free Krebs solution. The effects of two calcium antagonists, Nimodipine and Diltiazem were also studied. All the substances produced concentrationrelated contractions in normal Krebs solution. In the calcium free Krebs the responses to the non prostanoid spasmogens were almost completely abolished, whereas those to $\mathrm{PGF}_{2 \mathrm{a}}$, and U-46619 were only approximately $50 \%$ blocked. Concentration effect curves were then obtained in the presence of increasing concentrations of the two calcium antagonists respectively. The responses to 5-HT, NA, $\mathrm{KCl}$ and All were almost completely blocked; however, the contractions produced by the prostanoids were attenuated by only $50 \%$ at the highest concentrations. In contrast to these results, when applied after the "spasmogen", both Nimodipine and Diltiazem produced a concentration related relaxation of the sustained contractions produced all the agents. The results appear to have significant clinical applications. Thus the two calcium antagonists were only partially effective in preventing prostanoid induced contractions, but produced total reversal of sustained contraction irrespective of the spasmogenic agent.

CEREBRAL BLOOD FLOW AND NEUROLOGICAL OUTCOME: A CONTROLLED STUDY OF NIMODIPINE IN PATIENTS WITH SUBARACHNOID HAEMORRHAGE

EW Mee, DE Dorrance, D Low, G NeilDwyer, London

The authors had investigated the effect of Nimodipine, a calcium angagonist agent, upon the cerebral blood flow and outcome of patients suffering from subarachnoid haemorrhage. Seventy five patients had been entered into the double-blind placebo controlled prospective study, commencing within 96 hours of the haemorrhage. The xenon 133 inhalation method was used to measure cerebral blood flow daily for the 21 days of drug therapy. Outcome was assessed at three months. Twenty five patients were excluded from the analysis and the remaining 50 were divided equally between treated and control groups. Five patients in each group did not undergo operation. The active treatment did not produce serious complications; it was associated with a lowering of blood pressure by $5 \mathrm{mmHg}$ in the first day of treatment and with a rise in cerebral blood flow in patients whose treatment was commenced within 48 hours of haemorrhage. However, subsequently the drug treated patients had lower blood flows. In the patients treated with Nimodipine the result was considered "good" in 19 cases, "poor" in five cases, and one patient died. The corresponding figures in the control group were $13 ; 6$; and 6 . The authors concluded that the results showed a significant improvement in outcome as a result of the active treatment.

NEUROPSYCHOLOGICAL ASPECTS OF LONG TERM SURVIVAL IN TWO PATIENTS WITH MALIGNANT CEREBRAL GLIOMA

CA Prior, Swansea

The author, a senior clinical psychologist, described two adult patients, who were followed-up for 17 and 9 years, respectively after excision of glioblastoma multiforme of the left prefrontal area and whole-brain irradiation. Both patients made full neurological recoveries. The first, a shipwright, continued to work for over ten years, although his employer's and wife's reports indicated that he had become childish and unproductive in behaviour. The second patient, a housewife, had become passive and unable to carry out her role in household management, despite feeling guilt. Her husband had observed no change in her cognitive functioning or social behaviour. Serial CT scanning of both patients showed left frontal porencephaly, almost corresponding in size to a left frontal lobectomy in the first, but smaller in the second, who however, also showed ex-vacuo, general ventricular dilatation. In the first patient, a late increase in left perisylvian sulcal atrophy was accompanied by sensorimotor paresis of the right hand, and later, by an akinetic seizure for which a drug regime was commenced. Psychometric assessment of both patients indicated relatively little cognitive disturbance. The male patient (estimated premorbid IQ 96) obtained WAIS VIQ 88, PIQ 99 ten years after treatment, and WAIS VIQ 92, PIQ 103 after sixteen years. His Wechsler Memory $Q$ was 112 despite alleged forgetfulness. Language testing revealed impaired word fluency and, latterly verbal paraphasic errors occasionally in speech. The female patient (estimated premorbid IQ 112) showed some general intellectural deterioration: 1976, WAIS pre-operative VIQ 102, PIQ 105; WAIS VIQ 97, PIQ 99. There were no marked, focal features, and no detectable impairment of memory or linguistic functions. The loss primarily of self-organised, productive behaviour in both patients was taken to support the view that the prefrontal cortex essentially exerts an integrative influence at the highest level of behavioural organisation.

SKIN COVER: AN EXPANDING FIELD

MF Green, Swansea

The provision of adequate skin cover for the scalp is a problem encountered in both neurosurgery and plastic surgery. The author, who is a plastic surgeon, considered that the simple provision of skin grafts to denuded scalp is not satisfactory where the integrity of the cranial cavity may be lost, so that the need for flap closure had taxed him in numerous ways. Reconstructive surgeons had recently used increasingly complex flaps; these ranged from local adjacent scalp and aponeurosis to multiple stage procedures to carry distant skin flaps. The author reviewed two recent advances in the provision of scalp cover. The first was the advent of microvascular surgery; this had enabled the primary provision of scalp cover by revascularisation and re-implantation of the scalp or by the use of free vascular anastomosed omentum or muscle covered by split skin grafts or by musculo-cutaneous flaps. The second was the concept of tissue expansion as applied to the scalp; this ha enabled apparently new skin to be devel oped. These techniques had enabled bald are eas of the scalp, resulting from burns of $\overrightarrow{0}$ trauma, initially to be covered by split skifis graft, and then to be covered with scalp. The provision of locally available expanded skin ready for use enabled surgical procedures to take place where removal of tissue would have been compromosed by the quality of post operative skin cover. The author concluded that the provision of adequate skin cover to defects in the scalp had undergone major changes and that the development of new techniques and flaps had made possible procedures for problems previously unmanageable.

CHANGING PATTERN OF PITUITARY PERFUSION WITH DEVELOPMENT OF EXPERIMENTAL ANTERIOR PITUITARY TUMOUR PD Lees, HK Richards, S Perry, AHJ Lovick, JD Pickard, Southampton

The study was stimulated by the concept that changes in hypothalamic control of pituitary ademonata may reflect, in part, changes in the vascular supply of the anterior pituitary, In order to assess the overall pattern of perfusion throughout the gland

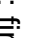
年 
the authors had used iodo $14_{c}$ antipyrine autoradiography to study Fischer 344 rats at varying times after they had been commenced on chronic oestrogen therapy, given in order to produce prolactin-secreting "tumours". With increasing size of the anterior pituitary, the pattern of blood flow changed from one of relatively homogenous perfusion to one of considerable heterogeneity which, after three weeks, was particularly marked in the periphery of the gland. In discussion the authors noted the problems of absolute measurement in different parts of the gland.

BLOOD FLOW IN ADENOHYPOPYSIS WITH OESTROGEN INDUCED PITUITARY ADENOMAS J Jakubowski, AA Kemeny, A Stawowy, WR Timperley, Sheffield

The mode of action on a pituitary tumour of agents such as bromocriptine is still not clear, but the possibility of a direct effect on vascularity of the adenohypophysis provided the basis for this study. The experiments concerned dynamic study of blood flow in the adenohypophysis of rats with a stilbestrol (DES) induced pituitary adenoma. Twenty female Fisher 344 rats comprised the control group; in 33 animals slow release silastic capsules containing DES were placed subcutaneously. Blood flow measurements and histological examination were carried out six weeks $(n=13), 9$ weeks $(n=12), 10$ weeks $(n=8)$ and 13 weeks ( $=5$ ) after implantation. The pituitary gland was exposed using a parapharyngeal route and two platinum wire electrodes were placed into visibly changed areas of adenohypophysis. The measurements of CBF were performed using the $\mathrm{H} 2$ clearance method. The following features were noted. Growth of the animals slowed down and their weight settled down to that of normal six weeks old. Blood pressure steadily increased from $100 \pm 15)$ to $126( \pm 16) \mathrm{mm}$ $\mathrm{Hg}$ by 13 weeks. The blood flow in the adenohypophysis was progressively reduced from $112 \pm 12 \mathrm{~mm} / 100 / \mathrm{min}$ in controls to $25( \pm 8) \mathrm{mm} / 100 / \mathrm{min}$ by the 13 weeks following implant of DES. The weight of the pituitary gland increased continuously reaching $101( \pm 36) \mathrm{mm} 10$ times that of the normal. Histological examination revealed that three out of 10 rats had developed an adenoma by six weeks and nine out of 10 by the tenth week. The adenohypophysis surrounding the adenoma became hyperplastic. "Vascular lakes" developed by the sixth week, which increased in size and frequency with time. Areas of tissue disruption by haemorrhage were seen in the late stages. The observed reduction in the blood flow in the adenohypophysis was explained by the development of "vascular lakes", resulting in stasis and possibly by compression of vessels by the increased size of the gland.

RADICAL TRANSTHORACIC SURGERY FOR THORACIC DISC PROTRUSIONS

GFG Findlay, Liverpool

The author commented that the removal of large calcified thoracic disc protrusions responsible for myelopathy remained a demanding and potentially dangerous procedure. The early results of laminectomy had produced poor results and the adaptation of the costotransversectomy approach by Hulme in 1960 was a dramatic improvement, but when dealing with very large protrusions this approach had limitations. Early experience with a transthoracic approach showed that the exposure of the disc fragment was facilitated but that excision through the confines of the disc space with only minimal bone removal could still be hazardous. The transthoracic approach was therefore extended to include partial corporectomies of the adjacent vertebral bodies prior to disc removal; this facilitated early dural exposure promimal and distal to the site of compression allowing a much safer mobilisation of the disc fragment. The resulting bone defect was made good with an iliac crest strut graft. The author reported on 20 patients encountered in a three year period. Of these 17 (age range 22-71 years) presented with central discs causing myelopathy, 12 with a level higher than D11/12. Eight patients were unable to stand and two had urinary retention. All 17 were treated as described above. Fifteen patients made a full recovery and were able to return to their premorbid level of activity. One patient improved to a lesser degree and the final patient remained unchanged. No patient was made worse. The author considered that the technique described offered an easier and safer method of treatment and allowed major neurological recovery. The addition of bone grafting did not cause problems, provided the surgeon was experienced in such techniques.

THE SURGICAL TREATMENT OF CERVICAL MYELOPATHY DUE TO SPONDYLOSIS AND DISC DEGENERATION

RV Jeffreys, Liverpool

The author presented a prospective study of the surgical treatment of cervical myelopathy due to spondylosis and disc protrusion. The study spanned 9.5 years
(1975-1984) and concerned 137 patient疋 There had been specific indications for choice of operation. Anterior (Cloward operation had been used to remove any anterior protrusion extending $4 \mathrm{~mm}$ or more beyond the vertebral body. Posterior lamp nectomy had been used for patients with multiple protrusions of $3 \mathrm{~mm}$ or less and with narrowing of the theco-periosteid diameter to less than $12 \mathrm{~mm}$, or with postrior protrusions only or with a congenitial narrow canal and superimposed spondylo without a major anterior protrusion. OTe hundred and twenty seven patients undeewent one operation only, 98 Cloward's a $\overline{\overline{\mathrm{m}}}$ 29 a laminectomy; 10 underwent two op ations, five had two Cloward's and five? Cloward and a laminectomy. Before opesation $55 \%$ of patients were so handicapped that they were dependent on others for daily living and $45 \%$ were handicapped in such way that they could manage personal feed, ing and hygiene but little else. The comptcation rate was $14 \%$ for the Cloward ope ation and $6 \%$ for laminectomy. At $6 \% 9$

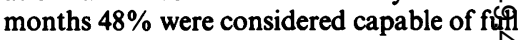
employment, $34 \%$ capable of light emplox ment, $17 \%$ were independent within the home and its environs and $1 \%$ who initiafy improved later deteriorated to dependent existence. The severity of the pre-operitive disability had a statistically adverse bearifg on outcome, but the length of history, myelographic findings and type of rological deficit did not. The author cluded that a defined policy of choøsing between anterior or posterior operatiog, according to the criteria described, had preduced results that were better than eith non-operative treatments or those obtainod by the employment of either operatif alone.

THE DISAPPEARING TUMOUR: AN UNUSUAT CASE OF PRIMARY CEREBRAL LYMPHOMA AN Jha, SS Pollock, PL Richardso Manchester

The authors reported a 13-year-old healt $\overline{\text { Ky }}$ Jewish boy who had presented in December 1983 with a short history of raised introcranial pressure, right facial weakness and papilloedema and which was shown on G scan and angiography to be due to a left frontal tumour. After dexamethasone, biopsy showed only reactive brain tissue: Repeat CT scan showed resolution of the mass but confirmed the correct location of the needle tracks. The patient remained wet until November 84 , when after a mild pyre ial illness, he developed unruly behaviow, 
wilful double incontinence, coprolalia and hypomania. These did not respond to haloperidol and a month later the patient developed a transient left sided ataxic nystagmus, a left hemiparesis, left upper quadrantanopia and an intermittent partial right third nerve palsy. A right frontal EEG focus was found but high resolution CT and magnetic resonance imaging each showed no evidence of a mass lesion. Extensive virological and immunological studies were unhelpful. In April 1985 he developed further features of raised intracranial pressure and CT showed a fresh, uniformly enhancing mass in the right posterior temporo-parietal area. At craniotomy a rubbery mass was partially resected which proved to be a primary $B$ cell IgM-kappa lymphoma. Following complete neuraxial radiotherapy and combination chemotherapy, there has been radiological shrinkage of tumour but no corresponding clinical improvement. The authors emphasised the diagnostic difficulties caused by the ability of a primary cerebral lymphoma to regress rapidly with complete clinical and radiological resolution. The case also showed the limitation of both CT scanning and magnetic resonance imaging in the diagnosis and monitoring of this condition.

CHANGES IN CEREBRAL BLOOD FLOW, BRAIN WATER AND INTRACRANIAL PRESSURE AFTER REMOVAL OF AN EXPERIMENTALLY PRODUCED MASS

EJ Sinar, AD Mendelow, GM Teasdale, Glasgow

The purpose of this study was to discover if changes in ICP, brain water and regional cerebral blood flow occurred after the removal of an intracerebral mass experimentally produced. A small microballoon had been inserted stereotactically into the right caudate nucleus of anaesthetised rats. It was inflated for ten minutes to a volume of $50 \mu \mathrm{l}$ and then deflated. In one group of seven animals the ICP was measured con-

tinuously with a contralateral ventricular catheter and brain water measured at the end of four hours using a microgravimetric method. In another group of six animals regional cerebral blood flow (CBF) was measured at the end of the four hour period using an autoradiographic technique with $14 \mathrm{C}$ iodoantipyrine (IAP). In each case the results were compared with a control group of sham-operated animals. Following inflation the ICP rose markedly, but returned to control values after deflation (table). There were no significant differences between the specific gravity measurements of the areas measured at 4 hours compared with controls. However, examination of the 14C-IAP autoradiographs revealed a marked reduction in CBF in the caudate nucleus on the side of the lesion when compared either with the sham group or with the contralateral side.

The authors concluded that ICP remained elevated for as long as the mass was present and rapidly returned to normal on its removal, also that there was not a measurable increase in water content at the end of four hours. On the other hand there was marked reduction in CBF on the side of the lesion, even four hours after the lesion had been removed.

COLLAGEN, AORTIC COMPLIANCE AND GENETIC PROBING IN SUBARACHNOID HAEMORRHAGE

G Neil Dwyer, FM Pope, AC Nichoolls, D Dorrance, P Narcisi, JR Bartlett, London In a previous study the authors had demonstrated that 13 of 24 patients with ruptured intracranial aneurysms had type III collagen deficiency. The methods they had used were time consuming, expensive and took a minimum of three months from the time of skin biopsy to the result. Recently they had studied Doppler shift continuous pulse wave ultrasound signals, whose velocity is directly related to the arterial compliance; the value

Table ICP and CBF after reversal of expanding lesion

\section{Lesion}

$6 \cdot 3 \pm 1 \cdot 4$

$14 \cdot 2+3 \cdot 0$

$5.6 \pm 0.5$

$5.9 \pm 0.6$

$5 \cdot 7 \pm 1.0$

$6 \cdot 1 \pm 0.8$

$C B F<20 \mathrm{ml} / 100 \mathrm{gm} / \mathrm{min}$

$\%$ Hemisphere
Right

$10 \cdot 0$

$\pm 6.0$
Left
$1 \cdot 7$
\pm 0.5
Right

1.9

$\pm 0.8$
Left

$2 \cdot 9$ of this measurement was that collagen is a $Z$ major constituent of arterial walls, so that $\stackrel{\mathbb{D}}{=}$ qualitative or quantitative alteration in its distribution might produce significant $\stackrel{\mathscr{C}}{=}$ changes in arterial distensibility. The aortico compliance was measured in 10 patients 0 with an intracranial aneurysm and whose type III collagen levels were known. Of four $\stackrel{\rho}{?}$ patients with a specific biochemical deficiency of type III collagen, three had. elevated aortic compliance and in total six of the 10 patients had elevated aortic compli- $\vec{P}$ ance. The study had been extended to othero patients with subarachnoid haemorrhage $\frac{\bar{\sigma}}{\sigma}$ and also their families. Twenty two of $30 \overline{\overline{\mathrm{s}}}$ patients had significantly elevated aortic $\overparen{\mathbb{Q}}$ compliance and $70 \%$ of their relatives had $\varrho$ raised compliance. Eight aneurysm patients had a normal compliance and $50 \%$ of the $\overrightarrow{0}$ relatives of these patients had elevated aortic ? compliance. Messenger RNA had been $\vec{\omega}$ examined in an aneurysm patient with type $\omega$ III collagen deficiency and was shown to be markedly reduced indicating that the patient's collagen deficiency was due to a genetic defect. The authors' conclusion was. that they had shown type III collagen deficiency in patients with ruptured intracranial aneurysms and they believed this to? be due to a genetic disorder.

MOYA MOYA DISEASE: THREE RECENT CASES뻴. WEST YORKSHIRE

PT Van Hille, MM Cameron, Wakefield $\stackrel{0}{-}$ Moya moya, meaning "like a puff of smoke", is a Japanese term used to describe the angiographic picture of profuse net-like anastomotic channels seen in association with stenosis or occlusion of the carotid@ artery at its bifurcation and hypoplasia of $\frac{}{\circ}$ the middle and anterior cerebral arteries. Of the 1000 cases reported, more than $90 \%$ had $\overrightarrow{\vec{O}}$ come from Japan and the authors therefore 3 described experience of three cases they had encountered in their area. Case 1 was a으. 58-year-old male who presented with a subarachnoid haemorrhage. An angiogram showed bilateral moya moya disease and an? aneurysm on the abnormal peripheral vessels. He was managed conservatively but a. second haemorrhage 2 months later killed $\varrho$ him. Case 2 was a 28 -year-old female with a coma producing subarachnoid hae-음 morrhage. CT showed intraventricular blood and angiography showed bilateralo moya moya, but no source for the haemorrhage. She remained well at 6 months' $N$ follow up. Case 3 was a 7-year-old male with a left hemiplegia after repair of an aortic coarctation. Three months later he suffered a further event which resulted in a right 
hemiplegia. CT showed bilateral infarcts and carotid angiograms demonstrated bilateral moya moya disease. He made good clinical progress after undergoing bilateral encephalomyosynangiosis. In discussion the authors commented that although the source of haemorrhage in moya moya disease was regarded as rupture of the abnormal vessels, the incidence of aneurysms with the disease was 5-6\%. The aneurysms were either normal saccular aneurysms on the circle of Willis (half occurring in the vertebrobasilar system) or so-called pseudoaneurysms, on the abnormal peripheral or basal vessels. They advised that once identified these aneurysms should be treated by operation. The ischaemic events were progressive and the surgical management was aimed at improving blood flow, by superficial temporal to middle cerebral artery anastomosis, encephalomyosynangiosis, or encephaloduro-arteriosynangiosis.

FLOW FLUORESCENCE CYTOMETRIC ANALYSIS OF DNA CONTENT IN 32 MENINGIOMAS COMPARED WITH CLINICAL AND HISTOLOGICAL RISK FACTORS AND HORMONAL RECEPTOR STATUS

RDE Battersby, JW Ironside, Sheffield

Meningiomas recur in a significant proportion of cases when after an apparently complete surgical removal has been accomlished; incomplete removal is invariably followed by an eventual recurrence. Some risk factors for recurrence have been identified, but prediction of recurrence is unreliable. The authors had used flow fluorescence cytometry (FFCM) to measure nuclear DNA distribution during the cell cycle to obtain an assessment of the proliferative activity of tumour cells, in the hope that this might provide a clue to the risk of recurrence. The nuclear DNA content of 32 menigiomas from 27 patients was measured in stored frozen tissue and in cell cultures derived from each tumour. When stored frozen tissue was examined 21 tumours had a "typical" DNA content (diploid cell line with small $G 2$ and $S$ phase peaks $(<20$ total), whereas 11 tumours had an "atypical" DNA content (aneuploid cells with multiple $G 1$ and $G 2$ peaks, or a diploid cell line with high G2 and S phase cells). Four tumours, each from a female patient, with an "atypical" DNA content in frozen tissue, had a "typical" DNA content in cell culture. The remaining tumours in cell culture retained the same SNA content as the frozen tissue. The results were compared with clinical and histological risk factors for recurrence, and with the hormonal receptor sta- tus of the tumour. The authors concluded that the FFCM technique was a rapid method of analysis, which might predict recurrence in meningiomas and might also indicate the likely response to treatments other than surgery, such as radiotherapy and possibly hormonal manipulation.

ADJUVANT CHEMOTHERAPY FOR MALIGNANT GLIOMA: IN VITRO AND IN VIVO EXPERIENCE WITH A COMBINATION OF PROCARBAZINE, CCNU AND VINCRISTINE

DGT Thomas, JL Darling, London

The authors described one hundred and forty six patients with malignant cerebral glioma (Kerhoham grades III and IV) who, following surgery, had been treated with adjuvant chemotherapy using procarbazine (PCB), CCNU and vincristine (VCR) and with whole head irradiation. Cell cultures were successfully prepared from 50 patients in the series and their senstivity to each cytotoxic drug was assessed in a microtitration assay using 35S-methionine incorporation. Cultures were designated sensitive or nonsensitive in vitro in comparison with a "training set" of 127 cases of malignant glioma previously investigated by cell culture. Those tumours whose cell cultures, ID50 (dose of drug causing $50 \%$ inhibition of protein synthesis, as measured by 35S-methionine uptake, in treated cultures compared with untreated cells of the same individual tumour) were below the median of the training set were designated sensitive and those whose ID50 were above the median were designated non-sensitive. In the 50 tested malignant gliomas 28 appeared sensitive and 22 non-sensitive to PCB and/or CCNU. The patients were followed clinically and by CT scan for periods of between 1 to 8 years, and the time to relapse determined. The relapse free interval (RFI) was associated significantly with the in vitro chemosensitivity to the two alkylating chemotherapy agents. PCB and CCNU ( $p<0.0001$ ), but not with sensitivity to the unrelated agent VCR. There was no difference in sex ratio, extent of operation, radiation dose and degree of steroid cover between sensitive, non-sensitive, and untested groups. Grade III tumours tended to be more sensitive than Grade IV tumours, and patients with chemosensitive tumours in vitro. Further statistical analysis, taking into account these prognostic factors, indicated an association between chemosensitivity in vitro and RFI. The 5 year RFI in the chemosensitive group of malignant gliomas was $27 \%$.
ASSESSMENT OF THE EFFECTIVENESS OFZ SURGERY IN THE TREATMENT OF SPASMODICC TORTICOLLIS: APPLICATION OF VIDEO-O GRAPHICS

RH Lye, GW Rogers, M Rootes, Maneٍ chester

In the authors' opinion existing methods provide an inadequate analysis of the globa政 disability of patients suffering from spasmodic torticollis. They emphasised the need $\overline{\mathbf{Z}}$ for objective evaluation of the effectiveness of treatment for movement disorders such ascos spasmodic torticollis, so that proper com=o parison could be made between the results $\frac{\sigma}{\sigma}$ of different managements. A relatively inex- $\overline{\bar{c}}$ pensive system was described which used pre and post-operative videotape recordings of patients undergoing surgical treatment for torticollis. A microcomputer digitised still $\overrightarrow{-}$ frames from the video recordings. The movement of a reference marker on the $\overrightarrow{\vec{\omega}}$ patient's face was then tracked by the computer thus yielding a graphic presentation of oscillation of the marker around a mean? position. In a pilot study, analysis of pre and post-operative recordings obtained from. two patients enabled assessment of the effectiveness of surgery in reducing the oscillation about the mean. There was good cor- 0 respondence between the results so obtained and the clinical impression of effectiveness of surgery. In addition $60 \frac{7}{0}$ graphic representation, results also we्किe presented in tabular format. This form $f \vec{c}$ documentation facilitated storage in patient's record. The authors proposed that. tabulation of results would provide an accurate comparison of the effectiveness of different methods of treatment. Limitations of the method and possible errors were discussed. For example, analysis is currently

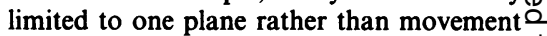
in three dimensions. Delays in digitising the $\overrightarrow{\vec{\sigma}}$ image and distortion or interference during 3 analysis of unstable video signals can introduce errors of approximately $10 \%$ in measurements of speed of oscillation. The authors emphasised that the problems encountered in this pilot study could be? overcome by simple modification of the $\frac{\sigma}{3}$ apparatus. They considered the technique? provided an objective means of assessing $\varrho$ movement disorders such as spasmodic tor- $\overline{3}$ ticollis, thus supplementing existing, moreo subjective methods.

INEXPENSIVE ULTRASOUND GUIDED BRAIN O BIOPSY

PG Richards, JM Rice Edwards, IM Maddison, London The use of ultrasound to aid the location of 
tumours for biopsy is a well established technique. Specialist machines are available for neurosurgical procedures but their use in Britain has not been widespread, mainly because of their cost. Portable ultrasound machines are readily available in obstetrics and in abdominal surgery and the authors had used an Aloka 710 Real Time Sector Scan ultrasound machine to image the brain and the spinal cord at operation. They reported that they had found it easy to locate small deep intracranial lesions and also to biopsy from various parts of a tumour because its wall and the centre were clearly visible on the machine. The machine was also used to locate solid lesions within the cystic spinal cord.

PROGNOSTIC FACTORS FOR MALIGNANT GLIOMAS

JN Wilden, JS Garfield, Southampton
A retrospective study was performed to identify prognostic features in 57 patients, selected for randomisation into the EORTC Trials. The criteria used to select patients were: supratentorial malignant glioma; over 16 years old; optimal removal of tumour; steroid therapy stopped within 10 days of surgery; post-operative Karnofsky index (Karnofsky et al 1984 Cancer 1:634-636) of $>70$; expected survival of 8 weeks or more and no other serious medical illnesses. All patients undertook a course of postoperative radiotherapy and some were treated with cytotoxic chemotherapy. Treatment started within 20 days of surgery. Using the Logrank and Breslow statistical tests, comparison was made between clinical features, CT scan, surgical procedures and survival or non-survival at 6 month epochs following surgery. The significant prognostic factors are shown in the table.
Table Prognostic factors for gliomas

Favourable Unfavourable

\begin{tabular}{|c|c|}
\hline $\begin{array}{l}\text { Less than } 50 \text { years } \\
\text { of age }\end{array}$ & Weakness \\
\hline Frontal location & $\begin{array}{l}\text { Visual field } \\
\text { abnormality }\end{array}$ \\
\hline $\begin{array}{l}\text { No surrounding low } \\
\text { density area on } \\
\text { scan }\end{array}$ & $\begin{array}{l}\text { Midline shift on CT } \\
\text { scan }\end{array}$ \\
\hline
\end{tabular}

The results illustrated that prognostic indices can be obtained for patients suffering from malignant gliomas. The authors pointed out that their important, but controversial finding that lobectomy increased survival was in broad agreement with other reports that local resection was better than biopsy. 